\title{
Étude des risques d'ébullition de sodium dans les réacteurs à neutrons rapides
}

\author{
Study of the sodium boiling hazard \\ in fast reactors
}

\author{
Jean Costa* \\ Chef de la Section de Thermohydraulique des Métaux Liquides \\ Centre d'Études Nucléaires de Grenoble
}

\section{Introduction : quelques rappels}

Rappels sur les conditions de fonctionnement d'un réacteur rapide (Chaumont et Al., 1978)

Dans le cœur d'un réacteur à neutrons rapides (ex. : Phenix ou Super-Phenix) en fonctionnement normal le fluide caloporteur du circuit primaire, le sodium, travaille à l'état liquide à des températures comprises entre $400^{\circ} \mathrm{C}$ et $600^{\circ} \mathrm{C}$. La température de saturation étant de lordre de $900^{\circ} \mathrm{C}$, l'ébullition du sodium est donc exclue dans les conditions normales de fonctionnement.

L'assemblage combustible est un boîtier hexagonal contenant, dans le cas de Super-Phenix, 271 aiguilles de $8,5 \mathrm{~mm}$ de diamètre, autour desquelles est enroulé un fil de $1,2 \mathrm{~mm}$ qui les maintient à un espacement régulier. Ce qui caractérise un tel assemblage est d'une part sa compacité, il ne reste environ que $30 \%$ de sa section pour le passage du sodium, et, d'autre part sa densité de puissance élevée, elle peut atteindre $500 \mathrm{~kW} / 1$ de cœur au centre.

\section{Rappels sur les dispositions de sûreté et les analyses d'accidents}

La sûreté de la centrale et la protection du public sont fondées (Noel et al., 1978) sur l'interposition en série de quatre barrières étanches entre le combustible radioactif et l'environnement. Ces barrières sont la gaine du crayon combustible, la cuve principale et le toit, l'enceinte de confinement primaire et enfin le bâtiment

(*) Centre d'Etudes Nucléaires de Grenoble

Département de Transfert en Conversion d'Energie

Service des Transferts Thermiques

Chef de la Section de Thermohydraulique des Métaux Liquides réacteur. Chacune de ces barrières donne lieu à des études très complètes sous l'angle de la prévention de sa détérioration, de la surveillance de son étanchéité et des actions de sécurité à engager en cas de défaillance.

Le niveau de redondance et la diversité des systèmes de sécurité sont tels que la probabilité de séquences accidentelles est extrêmement faible.

Dans les analyses de sûreté on étudie cependant des situations hypothétiques (Anselin et Penet, 1978) dans lesquelles il se produirait une élévation anormale de la température du cour suffisante pour conduire à la fusion des matériaux de structure et du combustible. Dans de tels scénarios le phénomène "ébullition du sodium" est présent et son rôle est extrêmement important car, par suite de couplages avec la neutronique, il influence le déroulement même de l'accident et conditionne ainsi la quantité globale d'énergie dégagée.

C'est pour ces raisons que l'ébullition du sodium a été considérée comme un risque et que ce phénomène donne lieu (en France et à l'étranger) à d'importantes études théoriques (développement de codes de calcul) et expérimentales (à caractère fondamental sur des circuits hors pile en complément d'expérience globales réalisées en pile).

On peut citer, à titre d'exemple, parmi les principaux types d'accidents retenus au niveau de l'analyse de sûreté :

- Des "accidents locaux", tels que le bouchage partiel ou total d'un pied d'assemblage (incident qui s'est produit en 1966 aux Etats Unis dans le réacteur FERMI), le bouchage de plusieurs sous-canaux dans la partie fissile. .

- Des "accidents globaux", tels que l'arrêt des pompes primaires sans chute des barres de sécurité, l'accident de réactivité.

Pour illustrer les "progrès récents accomplis dans l'étude des systèmes diphasiques" on ne traitera que le 
Tableau 1

Comparaison entre les propriétés physiques de l'eau et du sodium

\begin{tabular}{|c|c|c|c|}
\hline \multicolumn{2}{|c|}{ Propriétés physiques } & $\begin{array}{l}\text { Sodium } \\
\text { saturé à } 1 \text { b }\end{array}$ & $\begin{array}{c}\text { Eau } \\
\text { saturée à } 1 \text { b }\end{array}$ \\
\hline $\begin{array}{l}\text { Masse spécifique } \\
\text { Viscosité dynamique du liquide } \\
\text { Chaleur spécifique } \\
\text { Tension superficielle } \\
\text { Conductivité thermique } \\
\text { Temperature de saturation } \\
\text { Chaleur de vaporisation } \\
\text { Taux d'expansion } \\
\text { Viscosité dynamique de la vapeur } \\
\text { Nombre de Prandtl du liquide }\end{array}$ & $\begin{array}{l}\left(\mathrm{g} / \mathrm{cm}^{3}\right) \\
\text { (Poises) } \\
\left(\mathrm{J} / \mathrm{g} \cdot{ }^{\circ} \mathrm{C}\right) \\
(\text { dynes } / \mathrm{cm}) \\
\left(\mathrm{W} / \mathrm{cm}{ }^{\circ} \mathrm{C}\right) \\
\left({ }^{\circ} \mathrm{C}\right) \\
(\mathrm{J} / \mathrm{g}) \\
\text { (Poises) }\end{array}$ & $\begin{array}{c}0,74 \\
0,15610^{-2} \\
1,284 \\
118,7 \\
0,509 \\
880 \\
3877 \\
2776 \\
1,9710^{-4} \\
0,0039\end{array}$ & $\begin{array}{c}0,96 \\
0,28510^{-2} \\
4,21 \\
58,5 \\
0,68210^{-2} \\
99,63 \\
2258 \\
1630 \\
1,210^{-4} \\
1,76\end{array}$ \\
\hline
\end{tabular}

cas de l'ébullition au cours d'un arrêt des pompes sans chute des barres.

\section{Rappels sur les propriétés physiques du sodium et leurs conséquences (tableau 1)} $98^{\circ} \mathrm{C}$

Le sodium est un métal qui est liquide au-dessus de

Une comparaison de ses propriétés physiques à l'état liquide à $880^{\circ} \mathrm{C}$ avec celles de l'eau fait apparaître qu'il a presque la même densité, mais qu'il est environ 2 fois moins visqueux, que sa chaleur spécifique est à peu près 4 fois plus faible, que sa tension superficielle est 2 fois plus forte mais surtout que sa conductivité thermique est 100 fois meilleure. Sous 1 bar, à $880^{\circ} \mathrm{C}$ sa chaleur de vaporisation est 2 fois supérieure à celle de l'eau, de même que son taux d'expansion.

H. Mondin et R. Semeria (1969) ont étudié les conséquences prévisibles de ses propriétés physiques sur son comportement vis-à-vis de l'ébullition. Les principales sont :

- on peut s'attendre à ce que la surchauffe nécessaire au démarrage de l'ébullition soit 7 fois supérieure à celle de l'eau. Et en effet de nombreuses expériences ont mis en évidence de la surchauffe ;

- on peut prédire que la caractéristique interne d'un canal en ébullition aura une forme de courbe en $S$ très prononcée et qu'en conséquence un régime stable d'ébullition sera très difficile à obtenir (voir plus loin) ;

- on peut prédire également que la zone d'ébullition sous-saturée dans un canal chauffant sera extrêmement réduite comparée à celle qui peut exister avec de l'eau.

L'ébullition du sodium au cours d'un arrêt des pompes sans chute des barres ; le problème de la redistribution de débit

Par suite de caractéristiques propres aux réacteurs rapides développées en France, la baisse du débit de réfrigération du cœur, causée par l'arrêt des pompes primaires, est lente (fig. 1) ; elle est accompagnée d'une baisse de puissance (effets mécaniques, neutroniques) mais celle-ci, calculée avec des hypothèses pessimistes

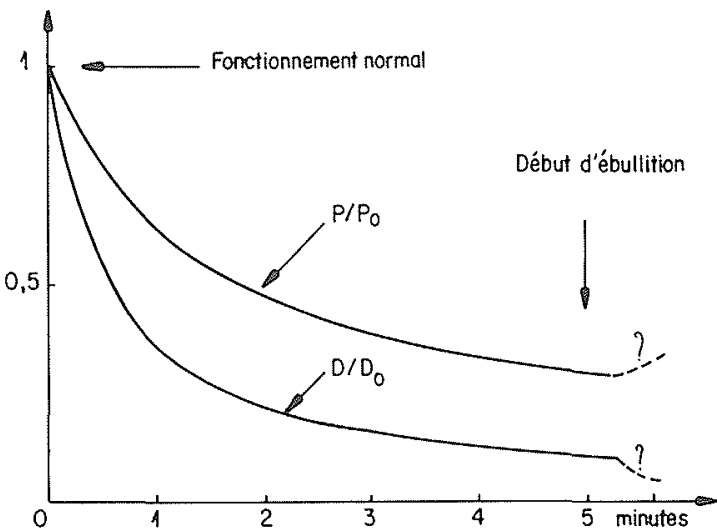

Figure 1. Décroissances $d u$ débit et de la puissance en cas d'arrêt de pompe sans chute des barres de sécurité.

n'est pas suffisante pour éviter l'apparition de l'ébullition au bout de plusieurs minutes.

Parce que la quantité globale d'énergie dégagée dans la suite de l'accident dépend fortement de la vitesse de vidange du cœur, le problème posé aux physiciens est de décrire le plus précisément possible le volume occupé par la vapeur de sodium dans chaque assemblage.

D'après la forme de la caractéristique interne d'un canal d'essais avec ébullition de sodium (courbe en $S$ mesurée par Charlety, 1971) et celle de la caractéristique externe au cours d'un arrêt des pompes, on peut prévoir l'apparition d'une instabilité de débit (Ledinegg, 1938) appelée "Redistribution de débit" (fig. 2).

La caractéristique interne est la courbe qui donne l'évolution de la perte de pression aux bornes du canal

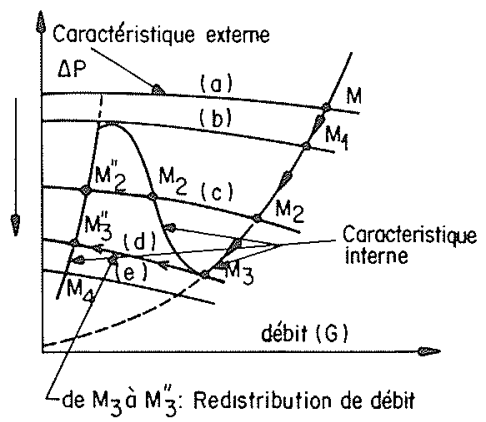

Figure 2. - Caractéristiques interne et externe pour un arrêt des pompes. 
d'essai en fonction du débit pour des conditions données de température d'entrée, pression de sortie et puissance injectée. Partant des débits élevés la décroissance de la perte de pression est celle correspondant au régime en simple phase liquide, la remontée de la courbe est causée par l'écoulement du fluide en ébullition (augmentation des termes $\Delta P$ frottement et $\Delta P$ accélération en double phase) et la courbe rejoint pratiquement la caractéristique du canal en phase vapeur.

$\mathrm{Au}$ cours du ralentissement des pompes la caractéristique externe évolue de (a) vers (e). Le point de fonctionnement en simple phase évolue de $M$ vers $M_{3}$. En $M_{3}$ l'ébullition apparaît et les nouveaux points de fonctionnement stables se situent en $M_{3}^{\prime \prime}$ ei $M_{4}$

La brusque réduction de débit de $M_{3}$ vers $M_{3}^{\prime \prime}$ est appelée "redistribution de débit". C'est une réduction incontrôlée du débit, irréversible car il faut remonter la caractéristique externe jusqu'en (b) pour revenir sur la partie simple phase de la caractéristique interne. En $M_{3}^{\prime \prime}$ le débit est très faible, le titre en vapeur est très élevé. En général le phénomène d'assèchement de la paroi chauffante apparait entre $M_{3}$ et $M_{3}^{\prime \prime}$.

Le problème, pour les analyses de sûreté des réacteurs rapides, consiste à décrire la vitesse à laquelle le débit passe de $M_{3}$ vers $M_{3}^{\prime \prime}$ ët quelle est au cours de ce transitoire la longueur de la zone en ébullition dans un assemblage.

\section{Tentative de résolution théorique}

On sait écrire les équations d'un modèle simple de double phase pour décrire l'ébullition du sodium en régime transitoire dans un canal et coupler ces équations avec les équations de la conduction dans les structures qui définissent le canal. Mais la résolution numérique est rendue extrêmement difficile par suite du caractère fortement non linéaire du couplage du taux de vide à la pression et du problème de la propagation des ondes de pression. A notre connaissance, aucune des tentatives entreprises pour résoudre théoriquement le problème n'a abouti à une solution satisfaisante.

\section{Approche expérimentale}

Des essais sur une maquette d'assemblage de réacteur en vraie grandeur sont difficilement concevables (coût, complexité, puissance nécessaire).

Dans le but de procéder du simple vers le compliqué, les premiers essais ont été réalisés en sodium sur une section d'essai ne comportant qu'une seule aiguille chauffante ${ }^{(1)}$. La géométrie monocanal n'est qu'une première approximation car on néglige les effets bidimensionnels (profils-radiaux de puissance, température et débit).

De plus, on n'a pas cherché à réaliser une simulation parfaite de la baisse progressive de la hauteur de refoulement des pompes. Au contraire on a centré l'objectif des essais sur la réalisation d'essais de redistribution de

(1) Chauffage électrique indirect - dimensions semblables à celles d'une aiguille combustible. Performances : température maxi : $1000^{\circ} \mathrm{C}$, puissance : $400 \mathrm{~W} / \mathrm{cm}$ - Technologie mise au point par le LATA (Laboratoire de Thermotechnique Avancée du Service des Transferts Thermiques.

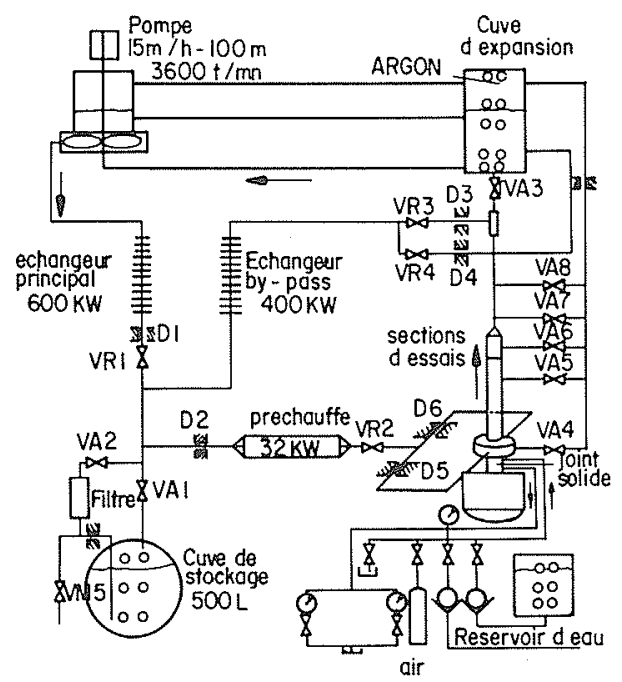

Figure 3. - Boucle CFNa II. Circuit d'essais en sodium.

débit les plus purs possible. Dans ce but, le point de départ de l'essai était un régime simple phase proche du minimum de la courbe et on déclenchait l'instabilité par un léger accroissement de la puissance, ce qui permettait plus de précisions qu'une légère modification de la caractéristique externe.

Les essais ont été réalisés sur plusieurs sections d'essais montées sur la boucle CFNa (fig. 3). Ce circuit, équipé d'une pompe de $15 \mathrm{~m}^{3} / \mathrm{h}$ et de $100 \mathrm{~m}$ de hauteur de refoulement permet d'imposer aux bornes de la section des caractéristiques externes variées grâce aux divers by-pass et vannes de réglage.

\section{Résultats et discussion (Schmitt, 1974)}

Un exemple typique de résultat est donné sur la figure 4. De nombreux autres résultats du même genre ont été obtenus pour des paramètres géométriques ou ther miques différents.

On distingue deux phases dans une telle redistribution de débit :

- la première au cours de laquelle le débit baisse lentement de sa valeur initiale à une valeur très faible (de $M_{3}$ à $M_{3}^{\prime \prime}$ sur la figure 2);

- la deuxième démarre avec l'apparition de fluctuations de la température de paroi correspondant à des assèchements successifs et est constituée d'un régime de

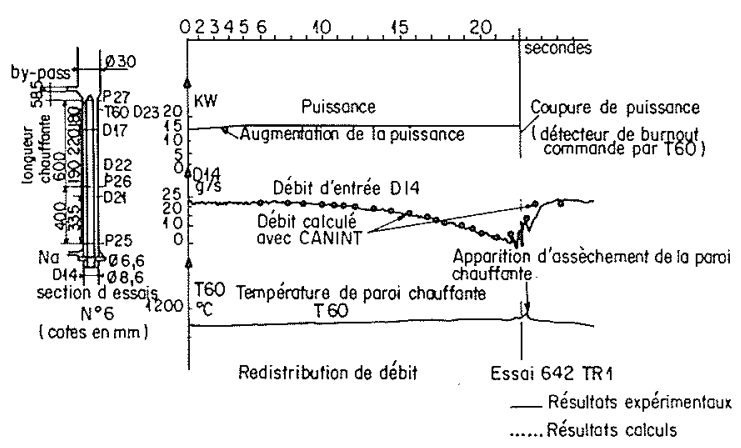

Figure 4. - Exemple de résultats d'essais. Comparaison expérience - Calcul. 
choucage. En général, au cours de ces oscillations le seuil de température maximum de l'expérience est atteint et on déclenche la coupure de la puissance. Après disparition de l'ébullition le débit reprend la valeur qu'il avait au début de l'expérience.

L'analyse de la série d'essais, effectués sur des sections d'essais différentes par la longueur des parties chauffantes, par la longueur des parties non-chauffantes à l'amont et à l'aval, par la valeur des paramètres puissance et température d'entrée nous a conduits à la conclusion que contrairement à ce que nous pouvions penser la redistribution de débit n'est pas contrôlée par l'hydrodynamique du système fluide mais plutôt par l'inertie thermique de la section d'essai. En effet les paramètres géométriques n'ont pas d'influence sur les vitesses de redistribution de débit et seule la puissance dissipée dans le canal ou la température d'entrée ont introduit des différences dans les résultats.

Dans le cas de nos expériences et dans le cas réacteur, la capacité calorifique des structures est grande devant celle du sodium, donc la puissance dissipée dans l'aiguille sert essentiellement à les échauffer et c'est ce qui contrôle la vitesse de redistribution du débit et qui explique que, dans le cas qui nous intéresse, elle soit lente.

Nous avons mis à profit le fait que ce transitoire était lent pour supprimer quelques termes transitoires des équations du modèle, ceux relatifs au fluide, pour ne garder que les termes transitoires de la conduction dans les parois. Le code simple CANINT ainsi obtenu (Schmitt, 1974) et d'autres plus élaborés, développés ultérieurement, ne présentent pas les difficultés mentionnées plus haut et permettent d'obtenir avec une bonne précision la variation du débit au cours des redistributions de débit (fig. 4) obtenues expérimentalement, et d'extrapoler les résultats aux problèmes du réacteur.

\section{Conclusions}

Par la présentation de ces études je pense avoir illustré des progrès récents accomplis dans le domaine de l'ébullition du sodium. La redistribution de débit n'est pas le seul problème, d'autres ont été ou sont étudiés, par exemple des excursions de puissance. Dans chaque cas les études expérimentales permettent de mettre en évidence les paramètres principaux qui gouvernent les phénomènes et ainsi d'aboutir à des codes de calculs construits sur des modèles simplifiés dont la résolution est plus aisée que les modèles complets.

Il convient de signaler que, dans notre domaine, la durée d'une étude comme celle-ci s'étale sur plusieurs années et que, en conséquence, l'expression "progrès récents" doit s'entendre comme progrès accomplis pendant les 5 dernières années.

Pour terminer, je voudrais mentionner un sujet de recherche sur lequel nous travaillons actuellement et pour lequel l'étude ci-dessus sert de base. Il s'agit de l'influence des hétérogénéités radiales de température, de débit et de puissance dans l'assemblage, sur le temps de vidange.

L'étude présentée a été menée sur une géométrie monocanal représentant de manière simplifiée un assemblage : elle a donc fourni une première approximation de

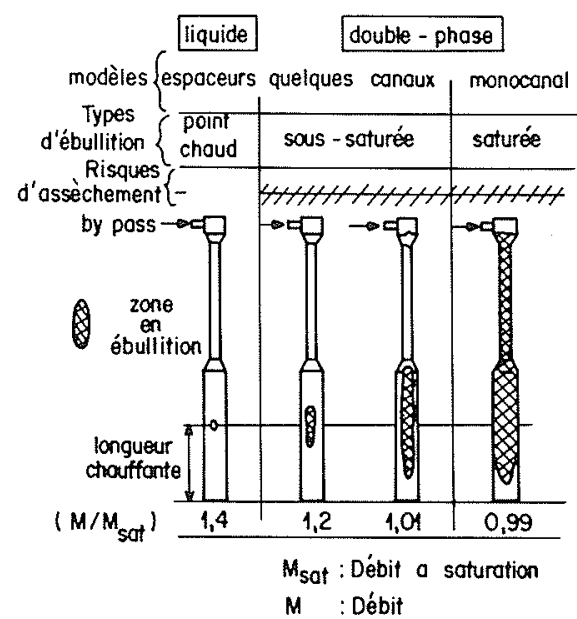

Figure 5. - Vidange d'un assemblage par ébullition. Redistribution interne de débit.

la vidange par ébullition. La question qui se pose désormais est de prendre en compte les effets bidimensionnels.

Sur la figure 5 nous avons porté des résultats qualitatifs (Menant, 1976) qui montrent un phénomène de redistribution interne entre les sous-canaux d'une grappe. Ce problème est de même nature que celui présenté ici, mais la différence réside dans le fait que les canaux communiquent entre eux. Nous essayons actuellement d'en saisir les principaux paramètres pour le modéliser. J'espère pouvoir, dans un temps relativement proche, faire part des progrès récents accomplis dans ce domaine.

\section{Références}

[1] ANSELIN F., PENET F. - Les Etudes et Recherches Relatives à la Sûreté du Projet Super-Phénix, Bulletin des Informations Scientifiques et Techniques du C.E.A., $\mathrm{n}^{\circ} 227$, pp. 119-139, 1978.

[2] CHARLETY P. - Ebullition du Sodium en Convection Forcée, Thèse de Docteur ingénieur - Faculté des Sciences de l'Université de Grenoble, 1971.

[3] CHAUMONT J.M., GRIGNON F., WALLET P. - Les Etudes et la Fabrication du Combustible de Super-Phénix, Bulletin d'Informations Scientifiques et Techniques du C.E.A., $\mathrm{n}^{\circ} 227$,pp. 63-80, 1978 .

[4] LEDINEGG M. - Instability of Flow during Natural and Forced Convection, Die Warme, 61, 8, pp. 891-898, AECTrans. (1954), 1938.

[5] MONDIN H., SEMERIA R. - Boiling and Two-Phase Flow of Sodium, Meeting of the European Two-Phase Flow Group, Karlsruhe, june 2-5, 1969.

[6] MENANT B. - Quelques Particularités des Ecoulements de Sodium Liquide et Bouillant dans des Grappes d'Aiguilles Chauffantes. Thèse de Docteur Ingénieur, Université Scientifique et Médicale de Grenoble et Institut National Polytechnique de Grenoble, 1976.

[7] NERSA. - Centrale Nucléaire de Creys-Malville (brochure).

[8] NOEL H., FRESLON H., LUCENET G. - Les Dispositions de Sûreté Relatives à la Centrale de Creys-Malville, Bulletin d'Informations Scientifiques et Techniques du C.E.A., $\mathrm{n}^{\circ} 227, \mathrm{pp} .81-98,1978$.

[9] SCHMITT F. - Contribution Expérimentale et Théorique d̀ l'Etude d'un type Particulier d'Ecoulement Transitoire de Sodium en Ebullition : La Redistribution de débit, Thèse de Docteur Ingénieur, Université Scientifique et Médicale de Grenoble, 1974. 


\section{Discussion}

Le Président remercie Monsieur COSTA de sa communication et ouvre la discussion

M. HENNIBICQ. - Le phénomène lié à la courbe en $\mathrm{S}$ que vous avez analysé est-il de même nature que les problèmes d'instabilité rencontrés dans les générateurs de vapeur des réacteurs à sodium.

M. $\operatorname{Cos} T A$. - La courbe en $\mathrm{S}$ que j'ai présentée sur la figure $\mathrm{n}^{\circ} 2$ est relative à un fluide à fort taux d'expansion (du sodium à la pression atmosphérique). Dans un générateur de vapeur la pression est très élevée, le taux d'expansion de l'eau est faible, il en résulte que la courbe en $S$ ne devrait plus présenter de minimum.

S'il y a des instabilités dans le générateur de vapeur, à mon sens elles ne sont pas dues au phénomène de redistribution de débit.

M. MONDIN. - On a constaté des instabilités dues aux ondes de densité dans les générateurs de vapeur.

M. LARMINAUX. - Le phénomène "courbe en S" se retrouve dans le générateur de vapeur, côté eau vapeur, de type circulation forcée. On règle le problème en ajoutant un diaphragme à l'entrée des tubes.

Le Président. - L'explication est peut-être dans la longueur du tube qui n'est pas négligeable dans les échangeurs. Le rapport de densité est un terme important, mais la longueur joue également.

Vous raisonnez avec des parties où l'ébullition est très limitée et votre zone de travail n'est pas la même. Mais je pense que le phénomène lié à la forme en $\mathrm{S}$ de la pression en fonction du débit à puissance donnée, est de même nature dans les deux cas.

M. DELHAYE. - Je voudrais poser une autre question relative au deuxième diagramme que vous avez présenté. Comment évolue le point de fonctionnement entre le point stable initi. et le nouveau point stable par rapport aux caractéristiques externes?

M. COSTA. - Il y a une variation lente ; aux bornes du canal, la pression qui existe est celle imposée par la caractéristique externe.

\section{Abstract \\ Study of the sodium boiling hazard in fast reactors}

In a fast reactor of the Super-Phénix type the sodium temperature lies between 400 and $600^{\circ} \mathrm{C}$. The saturation temperature, under the pressure of the core, being of the order of $900^{\circ} \mathrm{C}$, sodium boiling is not possible under normal operating conditions.

Despite the large safety margins and all the safeguard devices, hypothetical accident scenarios are taken into account in the safety studies. In these accidents the increase of the bulk temperature is large enough to reach the sodium boiling and materials melting limits. Because of positive reactivity feedbacks, the voiding rate of the core influences the energy deposition, therefore sodium boiling has been considered as a risk.

Typical hypothetical situations are :

- subassembly faults : inlet blockage, local blockage in the bundle,

- whole core accident : pump run-down without scram...

A description of sodium boiling phenomena during a slow pump rundown without scram has been chosen to illustrate the progress made in the study of two-phase systems.

The particular characteristics of the thermohydraulic studies presented are that they deal with a long pump coast-down time $(1 / 10$ of the nominal flow after about 10 minutes $)$ accompanied by a reduction of the power (about $1 / 3$ after 10 minutes) and involve a subassembly with wire-wrap spacers. Saturations conditions, based on bulk calculations, in the single channel approximation, are reached about 10 minutes after the onset of the pump run-down during a very slow transient so that stability considerations based on the Ledinegg criterion (flow excursion) are relevant.

As a quantitative description of this flow excursion was not available a special program was undertaken to understand it and to get experimental data to validate computer codes.

From the series of experiments performed in sodium in the single channel approximation, covering a wide range of parameters, different heated lengths, downstream geometries, and power levels, it can he concluded that the flow excursion is a rather slow process, lasting for a period of the order of several seconds. Two distinct phases can be observed :

- In the first phase, there is a progressive voiding of the

channel accompanied by a quiet boiling regime (bubbly flow),

- In the second phase, starting with the local dry-out of the pin there are expulsions (chugging).

The main reason for the rather slow decrease of the flow during the flow excursion is that the thermal inertia of the structural material and the pins is large compared to the thermal inertia of the sodium.

Although great care has been taken in the definition of the characteristics of the single channel test section a major uncertainty still remains as to how far the results obtained in such a simplified geometry are representative of a subassembly. A reply will be given by an on going program on small bundles. 